\title{
Secrets of RNA granules
}

A surprising hydrogel formation promises insight on prions and RNA transport.

A hunt for the protein targets of biologically active small molecules has instead revealed how certain membrane-free cellular structures might form. Following up on reported work, researchers led by Steven McKnight of the University of Texas Southwestern Medical Center began investigating an isoxazole identified for its ability to cause stem cells to differentiate.

Though the effects of this molecule were known, its protein targets were not. The researchers attached a biotin tag to the isoxazole, planning to fish the tag from mouse cell lysates and identify any attached proteins. But adding the biotinylated molecule (dubbed 'b-isox') to the cell lysates produced a precipitate that seemed to comprise a special class of proteins, which the team decided to explore further.

When greater amounts of b-isox were added to cell lysates, the amount of precipitated proteins also increased, yet the staining pattern of soluble proteins in the supernatant remained the same, recalls Masato Kato, a biochemist at UT Southwestern who led part of the study. "This suggested that the proteins precipitated by b-isox are rare proteins, and [that] there should be a selectivity mechanism for b-isox precipitation."

Analysis showed that the precipitated proteins consisted of hundreds of RNAbinding proteins, including many that are found in RNA granules, large complexes of RNA and protein which transport RNA to specific sites in a cell. Experiments using lysates treated with RNases showed that precipitation happened even without RNA, so the researchers began analyzing protein sequences and making recombinant proteins to understand how the precipitate might form (Kato et al., 2012).

Many of the precipitated proteins consisted of only two functional domains: one associated with RNA binding and another represented by highly repetitive regions with low complexity. Though others had proposed that such low-complexity (LC) regions might be important for proteinprotein interactions and transcription factor binding, it was unclear what mechanisms might be at work, says Kato.

After studies showing that the LC domains of several RNA-binding proteins were necessary and sufficient for precipitation, the researchers focused on the protein FUS (fused in sarcoma). They observed that high concentrations of this protein without b-isox could form hydrogels and that the hydrogel could bind other RNA-binding proteins with modest to moderate avidity, with retention attributed solely to the LC domains. A second protein, HNRNPA2-which, like FUS, is found in RNA granulesalso formed a hydrogel. X-ray diffraction studies showed that the hydrogels formed from either protein are composed of fibers. However, unlike for the pathogenic fibers associated with amyloid plaques and prion diseases, the formation of these fibers is reversible.

Assuming that what happens in vitro also happens in cells, the researchers postulate that this phenomenon-the polymerization of certain proteins into amyloid-like fibers-might be used as a cellular mechanism to form and reform protein and RNA clusters in subcellular domains. In fact, the researchers showed that when LC domains of FUS were phosphorylated, they were less capable of forming hydrogels, an observation that may point to how cells control the assembly and disassembly of RNA granules in appropriate parts of the cell.

Next, the researchers turned their attention to the RNA caught within these b-isox precipitates and hydrogels (Han et al., 2012). They treated mouse brain extracts and human cell-culture isolates with b-isox and then isolated and sequenced the associated RNAs.

Both b-isox precipitates and FUS hydrogels captured the same sorts of mRNAs. Captured transcripts were lon-

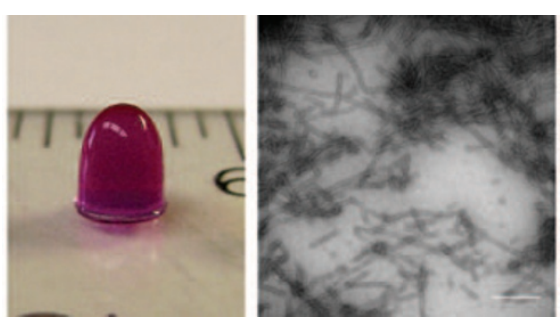

Image of a hydrogel made of proteins with short, highly repetitive regions (left) and image of amyloid-like fibers found in the hydrogel (right). Scale bar, $500 \mathrm{~nm}$. Images courtesy of M. Kato.

ger than uncaptured ones, particularly in their untranslated regions. The captured transcripts were also more likely to be those found in neuronal granules and to contain binding sites for granuleassociated proteins. Furthermore, captured mRNAs most frequently encoded proteins related to cell adhesion and axon guidance, functions that often depend on localized translation.

Currently, Kato and other researchers are analyzing different tissues and species as well as proteins found in different types of granules. The goals are to gather evidence that fiber polymerization of proteins with LC regions causes granule formation inside cells and to understand how widespread the mechanism might be. "For a hundred years, people have known about these non-membrane bound granules," says McKnight, "but the underlying architecture of how they form has been mysterious. If we're right, it's potentially really cool." Eventually these hydrogels could provide cell-free ways to study prions, RNA granules and other membranefree structures.

\section{Monya Baker}

\section{RESEARCH PAPERS}

Han, T.W. et al. Cell-free formation of RNA granules: bound RNAs identify features and components of cellular assemblies. Cell 149, 768-779 (2012).

Kato, M. et al. Cell-free formation of RNA granules: low complexity sequence domains form dynamic fibers within hydrogels. Cell 149, 753-767 (2012). 